\title{
Article \\ Overexpression of Orange Gene (OsOr-R115H) Enhances Heat Tolerance and Defense-Related Gene Expression in Rice (Oryza sativa L.)
}

\author{
Yu Jin Jung ${ }^{1,2,+}$, Ji Yun Go ${ }^{1,+}$, Hyo Ju Lee ${ }^{1}$, Jung Soon Park ${ }^{1}$, Jin Young Kim ${ }^{1}$, Ye Ji Lee ${ }^{1}$, Mi-Jeong Ahn ${ }^{3}{ }^{1}$, \\ Me-Sun Kim ${ }^{4}$, Yong-Gu Cho ${ }^{4}\left(\mathbb{D}\right.$, Sang-Soo Kwak ${ }^{5}$ (D) , Ho Soo Kim ${ }^{5}$ and Kwon Kyoo Kang ${ }^{1,2, *(D)}$ \\ 1 Division of Horticultural Biotechnology, Hankyong National University, Anseong 17579, Korea; \\ yuyu1216@hknu.ac.kr (Y.J.J.); rhwldbs60@naver.com (J.Y.G.); ju950114@naver.com (H.J.L.); \\ kgfood1@hanmail.net (J.S.P.); ajsw11202@naver.com (J.Y.K.); lyj7776@naver.com (Y.J.L.) \\ 2 Institute of Genetic Engineering, Hankyong National University, Anseong 17579, Korea \\ 3 College of Pharmacy and Research Institute of Life Sciences, Gyeongsang National University, \\ Jinju 52828, Korea; amj5812@gnu.ac.kr \\ 4 Department of Crop Science, Chungbuk National University, Cheongju 28644, Korea; \\ kimms0121@cbnu.ac.kr (M.-S.K.); ygcho@cbnu.ac.kr (Y.-G.C.) \\ 5 Plant Systems Engineering Research Center, Korea Research Institute of Bioscience and \\ Biotechnology (KRIBB), Daejeon 34141, Korea; sskwak@kribb.re.kr (S.-S.K.); hskim@kribb.re.kr (H.S.K.) \\ * Correspondence: kykang@hknu.ac.kr; Tel.: +82-31-670-5104 \\ + These authors contributed equally to this work.
}

\section{check for} updates

Citation: Jung, Y.J.; Go, J.Y.; Lee, H.J.; Park, J.S.; Kim, J.Y.; Lee, Y.J.; Ahn, M.-J.; Kim, M.-S.; Cho, Y.-G.; Kwak, S.-S.; et al. Overexpression of Orange Gene (OsOr-R115H) Enhances Heat Tolerance and Defense-Related Gene Expression in Rice (Oryza sativa L.). Genes 2021, 12, 1891. https://doi.org/ 10.3390/genes12121891

Academic Editors: Kejian Wang and Bing Yang

Received: 24 September 2021

Accepted: 25 November 2021

Published: 26 November 2021

Publisher's Note: MDPI stays neutral with regard to jurisdictional claims in published maps and institutional affiliations.

Copyright: (c) 2021 by the authors. Licensee MDPI, Basel, Switzerland. This article is an open access article distributed under the terms and conditions of the Creative Commons Attribution (CC BY) license (https:/ / creativecommons.org/licenses/by/ $4.0 /)$.

\begin{abstract}
In plants, the orange (Or) gene plays roles in regulating carotenoid biosynthesis and responses to environmental stress. The present study investigated whether the expression of rice Or $(\mathrm{OsOr})$ gene could enhance rice tolerance to heat stress conditions. The OsOr gene was cloned and constructed with OsOr or OsOr-R115H (leading to Arg to His substitution at position 115 on the OsOr protein), and transformed into rice plants. The chlorophyll contents and proline contents of transgenic lines were significantly higher than those of non-transgenic (NT) plants under heat stress conditions. However, we found that the levels of electrolyte leakage and malondialdehyde in transgenic lines were significantly reduced compared to NT plants under heat stress conditions. In addition, the levels of expression of four genes related to reactive oxygen species (ROS) scavenging enzymes (OsAPX2, OsCATA, OsCATB, OsSOD-Cu/Zn) and five genes (OsLEA3, OsDREB2A, OsDREB1A, OsP5CS, SNAC1) responded to abiotic stress was showed significantly higher in the transgenic lines than NT plants under heat stress conditions. Therefore, $\mathrm{OsOr}-\mathrm{R} 115 \mathrm{H}$ could be exploited as a promising strategy for developing new rice cultivars with improved heat stress tolerance.
\end{abstract}

Keywords: transgenic rice; heat tolerance; OsOr-R115H; gene expression

\section{Introduction}

Global warming is expected to significantly reduce crop yields due to high temperatures that negatively affect plant growth and development. Thermal stress can eventually lead to cell damage and death in plants due to accumulation of excess reactive oxygen species (ROS), membrane lipid peroxidation and metabolic disturbance [1,2]. Under elevated temperature, plants have shown physiological symptoms such as scorching of leaves and stems, leaf abscission and senescence, shoot and root growth inhibition or fruit damage, which lead to a decreased productivity and quality of crops [3]. Until now, numerous heat stress defense genes have been reported to mainly encode signaling molecules such as mitogen activated protein kinase (MAPK) cascades, cellular protective enzymes such as peroxidase (POD), transcription factors such as heat shock transcription factors (HSFs), and cellular stress proteins such as heat shock proteins (HSPs) [4-7]. These genes have been reported to protect plant cells by enhancing resistance with maintaining 
ROS homeostasis in the cell or regulating various genes on the signal pathway under thermal stress $[6,7]$. It was reported recently that the function for carotenoids relates to the response of plants to environmental stress [4,5]. In addition, reactive oxygen species (ROS) generated under stress conditions oxidizes carotenoids and converts into various oxidation products including aldehydes, ketones, endoperoxides, and lactones [4]. This demonstrates that carotenoid accumulation and potential signaling in plant cells plays a positive role in heat stress response. The Orange $(\mathrm{Or})$ gene encoding a cysteine-rich DnaJ protein has been reported to regulate carotenoid accumulation and abiotic stress resistance in various plant species [8-16]. The Or protein functions as a holdase chaperone, a post-transcriptional regulator of phytoene synthase (PSY), and a rate-limiting enzyme on the carotenoid biosynthetic pathway [12,17]. Moreover, Or protein has been reported to interact with oxygen-evolving enhancer protein 2-1 (PsbP) in the chloroplast and protect it from heat-induced denaturation $[17,18]$. An allele carrying a single nucleotide polymorphism (SNP), in which the 96th amino acid of the Or protein substituted arginine (Arg) to histidine (His), is associated with high-carotene accumulation in melon [9]. In addition, overexpression of AtOr protein (Q8VYD8; AtOr-R96H) leads to high level accumulation of carotenoids in Arabidopsis and tomatoes (Solanum lycopersicum) $[13,19]$. In the sequence comparison of the $\mathrm{Or}$ gene between the low-carotenoid and high-carotenoid lines in carrots (Daucus carota), SNP substituted from $\mathrm{T}$ to $\mathrm{C}$ has been identified, and it is reported that a codon TTG (leu) is changed to TCG (ser), resulting in high carotene accumulation [20]. In a recent experiment, we have found out that the site-directed mutagenesis of IbOr gene in sweet potato (Ipomoea batatas [L.] Lam), leading to Arg to His substitution at position 96 (based on the CmOr protein) in the IbOr protein (IbOr-R96H), showed significantly higher carotenoid contents and enhanced environmental stress tolerance [21,22]. In this study, to understand the physiological role of OsOr gene in rice, the carotenoid content and abiotic stress tolerance were investigated in overexpressing plants, OsOr-WT and OsOr-R115H. Our results show that in the overexpressing plants, $\mathrm{OsOr}$-WT and $\mathrm{OsO}-\mathrm{r}-\mathrm{R} 115 \mathrm{H}$, carotenoid content was not changed compared to NT plants, but heat stress tolerance was enhanced. In addition, these lines showed a high level of transcription of genes related to ROS scavenging enzymes under stress conditions. Interestingly, the OsOr-R115H transgenic line showed lower malondialdehyde (MDA), lower relative electrolyte leakage (REL), and higher proline content in leaves compared with NT plants.

\section{Materials and Methods}

\subsection{Growth Conditions and Stress Treatments}

Dongjin (Oryza sativa L.ssp. japonica) was used as a wild-type, cultivated in greenhouse facilities at Hankyong National University, Korea, as described by Jung et al. [23]. The expression levels of $\mathrm{OsOr}$ were investigated in roots, leaves, stems, panicles, and immature seeds at 7 days after pollination in Dongjin cultivar. The expression pattern of the OsOr in response to abiotic stresses including $48{ }^{\circ} \mathrm{C}$ heat, $200 \mathrm{mM} \mathrm{NaCl}$, and $20 \%$ PEG6000 drought $100 \mu \mathrm{M} \mathrm{GA}_{3}, 100 \mu \mathrm{M} \mathrm{ABA}$, and $4{ }^{\circ} \mathrm{C}$ cold was monitored as previously reported by Park et al. [17]. Abiotic stress treatments were performed for 0 h, $3 \mathrm{~h}, 6 \mathrm{~h}, 12 \mathrm{~h}$, and $24 \mathrm{~h}$, and then samples were collected and stored at $-80^{\circ} \mathrm{C}$. All experiments included three biological replicates.

\subsection{Gene Cloning and Generation of Transgenic Rice}

The full-length cDNA of OsOr (Os02g0651300) was amplified with specific primer sets for RT-PCR (Supplementary Table S1). The PCR product was cloned into pMD18-T vector (TAKARA, Seoul, Korea) and sequenced. Site-directed mutagenesis was performed to obtain point mutants by substituting $\mathrm{H}$ for the 115th amino acid $\mathrm{R}$ of the OsOr gene as previously described by Kim et al. [22]. Briefly, OsOr-R115H, which replaced the ORF (Open Reading Frame) (OsOr-WT) and major SNPs of the OsOr gene, was subcloned downstream of the binary vector pGWB5 expressed by the cauliflower mosaic virus (CaMV) $35 \mathrm{~S}$ promoter, respectively. The constructed vector was transformed into Agrobacterium tumefaciens 
strain EHA105, which was introduced by infection with rice embryogenic callus, as previously described [24]. The transformed callus and plants were selected using $6 \mathrm{mg} / \mathrm{L}$ phosphinothricin and confirmed by PCR analysis, as previously reported [24]. Positive transgenic plants were first screened by PCR, and then further confirmed by TaqMan PCR to selection of single copy insertion of T-DNA and qPCR analysis to determine the expression of the transgene. In addition, the transgenic homo lines of $\mathrm{T}_{3}$ generation were used for the analytical experiment.

\subsection{Phenotype Analysis}

Pollen viability was evaluated as previously described [25] from transgenic plants with OsOr gene and wild-type plants at flowering time for pollen germination analysis. Nikon SMZ800N microscope (Nikon Metrology Inc., Seoul, Korea) was used to observe pollen germination. The amylose content of whole grain was measured using the AMYLOSE / AMYLOPECTIN kit (Megazyme Ltd., Bray, Ireland) according to the manual provided by the manufacturer. The morphology of starch grains was observed by scanning electron microscopy (SEM). Images were captured using a Quanta FEG 450 instrument (ZFE, Graz, Austria). Xanthomonas oryzae pv. oryzae (Xoo) inoculation and determination of bacterial populations were evaluated as previously described $[26,27]$ for a wild-type plant and transgenic plants. In addition, 30 plants were randomly selected from each of the transgenic lines and NT plants, and the seed setting rate, pollen fertility, 1000-grain weight, and yield per plant were investigated.

\subsection{Analysis of Carotenoid Contents}

Carotenoids were extracted from rice leave tissues using $0.01 \%$ solution of butylatedhydroxytoluene in acetone and analyzed using an Agilent 1100 HPLC system (HewlettePackard, Palo Alto, CA, USA) according to the method described by Lim et al. [28].

\subsection{Analysis of Heat Tolerance of Transgenic Rice}

To induce heat stress, leaf discs were excised from the fourth leaves of 2-week-old transgenic and NT rice plants and treated to high temperature $\left(48^{\circ} \mathrm{C}\right)$ in the dark for $24 \mathrm{~h}$.

To measure tissue levels of $\mathrm{H}_{2} \mathrm{O}_{2}$ and visualize the degree of damage caused by heat stress, leaf discs were immersed in 3,3'-diaminobenzidine (DAB) solution $(1 \mathrm{mg} / \mathrm{mL}$, $\mathrm{pH}$ 3.8) for $5 \mathrm{~h}$ at $25^{\circ} \mathrm{C}$ under continuous light, as described previously [18]. To measure the extent of cellular damage or membrane disruption, ion leakage was quantified using an ion conductivity meter (MTD, Schwerzenbach, Switzerland). For quantitative analysis of total area of DAB staining region, images were processed using ImageJ.

\subsection{Physiological Parameters Measurements of Transgenic Rice}

To investigate whether the transgenic lines can be used as oxidative stress biomarkers, the relative electrolyte leakage (REL), proline, and malondialdehyde (MDA) content were detected based on the previously described methods. Briefly, $500 \mathrm{mg}$ of rice leaves were pulverized in $2 \mathrm{~mL}$ of the chilled reagent $(0.25 \%(w / v)$ thiobarbituric acid in $10 \%(w / v)$ trichloroacetic acid). The extracts were treated at $100{ }^{\circ} \mathrm{C}$ for $30 \mathrm{~min}$, cooled at room temperature, and centrifuged at $12,000 \times g$ for $15 \mathrm{~min}$. The absorbance of the supernatant was measured at 450,532, and $600 \mathrm{~nm}$. The MDA content was calculated according to the formula: $6.45 \times\left(\mathrm{OD}_{532}-\mathrm{OD}_{600}\right)-0.559 \times \mathrm{OD}_{450}$ [29-31].

\section{7. $q R T-P C R$ Analysis}

Total RNA was isolated from different tissues of rice plants using a RNeasy plant mini kit (Qiagen, Seoul, Korea, www.qiagen.com) respectively, and single-strand cDNA was synthesized with random oligonucleotides using a reverse transcription system (Bioneer, www.bioneer.co.kr, E-3122 (M-MLV Reverse Transcriptase) based on a previously reported method [18]. Primers used in the experiment are listed in Supplementary Table S1. qRTPCR analysis was performed according to the manufacturer's instructions using Light- 
CyclerR 480 Real Time PCR system (Roche, CA, United States). "Relative expression" levels were calculated using the $2^{-\Delta \Delta C T}$ method, and OsActin (Q10DV7) was used as a reference gene in rice. All experiments included three biological replicates, each with two technical replicates.

\subsection{Statistical Analysis}

Data collected in this study were analyzed by one-way analysis of variance (ANOVA) using the Statistical Analysis System (SAS version 9.4). Values are mean \pm SE $(n=3)$ and statistical significance was set to $p<0.05$ according to Duncan's multiple range test [32].

\section{Results}

\subsection{Expression Profile of OsOr Gene}

The full-length cDNA of OsOr was cloned into PMD18-T vector after PCR analysis using gene specific primer sets, and confirmed by sequencing. This gene consists of a $996 \mathrm{bp}$ ORF encoding a putative protein of 332 amino acids, and GenBank's accession number is AK099767 (Supplementary Figure S1A,C). The OsOr protein had an estimated molecular mass of $34.3 \mathrm{kDa}$ and pI of 8.36 (Supplementary Figure S1C). The genetic similarity between the sequences of OsOr and Or genes of various plant species showed the highest homology with the Or gene of morning glory (Ipomoea nil) (TA6874_35883) and was a 97\% identity at the amino acid level (Supplementary Figure S1B). In addition, OsOr showed 73-80\% sequence homology between several plant-derived Or genes, including the putative $\mathrm{Or}$ genes of tomato (L. esculentum), grape (Vitis vinifera), Arabidopsis thaliana (At5g61670), and cauliflower (B. oleracea var. botrytis) (Supplementary Figure S1C). The domain annotation of the OsOr protein contained a motif with two transmembrane domains, a plastid-targeting

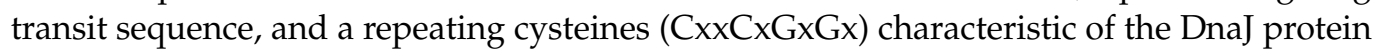
known as a chaperone. To determine the expression pattern of OsOr in rice, we analyzed the expression profile of OsOr gene in roots, stems, leaves, panicles, and immature seeds using qRT-PCR. The results showed that OsOr gene was expressed in all tissues tested, and this gene expression was highly detected in stems and lowly detected in immature seeds (Figure 1A). In addition, we investigated the expression pattern of the OsOr gene in response to $\mathrm{H}_{2} \mathrm{O}_{2}, \mathrm{PEG}$, heat, cold, $\mathrm{ABA}$, and $\mathrm{GA}_{3}$ stresses. The results suggested that $\mathrm{H}_{2} \mathrm{O}_{2}$, PEG, heat, and cold stresses except $\mathrm{GA}_{3}$ stress induced the expression of OsOr at all treatment time points (Figure 1B). Under $\mathrm{H}_{2} \mathrm{O}_{2}, \mathrm{PEG}$, and cold stress conditions, the expression level of $\mathrm{OsOr}$ was rapidly induced from the early stage and showed the plateau expressions for 3-24 $\mathrm{h}$ following the treatment. Under heat stress condition, the expression level of $\mathrm{OsOr}$ was rapidly induced, and gradually increased to $24 \mathrm{~h}$ of treatment. In addition, the cold stress condition slightly significantly induced the expression of $O s O r$, but the $\mathrm{GA}_{3}$ stress conditions did not show significant difference in the OsOr expression level. Therefore, the results of expression profile for OsOr indicated that OsOr gene might be important for plant tolerance against $\mathrm{H}_{2} \mathrm{O}_{2}, \mathrm{PEG}$, heat, and cold stresses.

\subsection{Phenotypic Analysis of Transgenic Rice}

To further study the function of the OsOr gene, we constructed $\mathrm{OsOr}-\mathrm{WT}$ and $\mathrm{OsOr}-\mathrm{R} 115 \mathrm{H}$ under the control of the CaMV $35 \mathrm{~S}$ promoter and introduced them in rice embryogenic callus using an Agrobacterium-mediated method (Figure 2A). To select transgenic plants with a single copy gene, ten $\mathrm{T} 0$ transformants with OsOr-WT and $\mathrm{OsOr}$ - $\mathrm{R} 115 \mathrm{H}$ were tested through PCR analysis on whether T-DNA was inserted as well as a TaqMan PCR analysis. Four independent T3 homozygous transgenic lines, \#7 and \#9 for OsOr-WT, and \#6 and $\# 10$ for OsOr-R115H, were selected and used for subsequent analysis (Supplementary Figure S2; Figure 2B). 


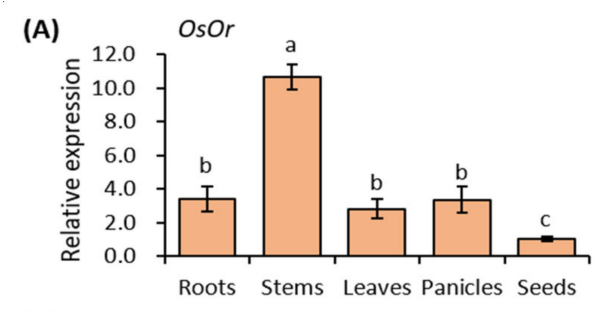

(B)
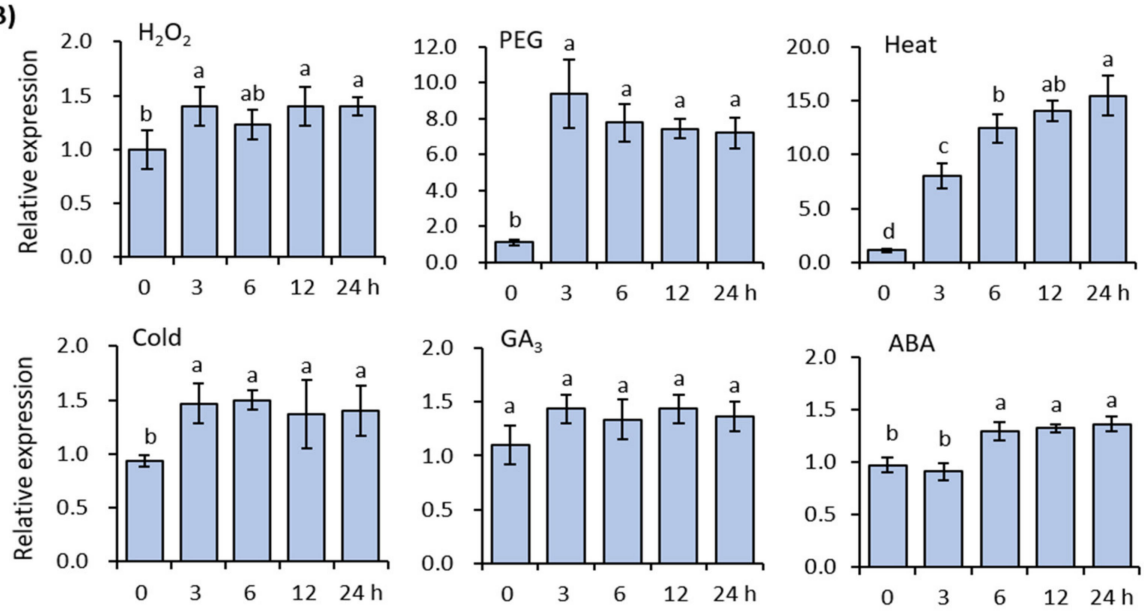

Figure 1. Expression analysis of the OsOr gene in $\mathrm{T}_{3}$ generation. (A) qRT-PCR analysis of OsOr gene expression in various tissues. Bars show means $\pm \mathrm{SD}$ with three biological replicates. (B) OsOr expression analysis (qRT-PCR) in leaves of 2-week-old rice seedlings subjected to $\mathrm{H}_{2} \mathrm{O}_{2}, 20 \%$ PEG 6000, heat, cold, ABA and $\mathrm{GA}_{3}$ treatments, respectively. Bars show standard deviations of the replicates. Data are means $\pm \mathrm{SE}(n=3)$. Different letters above the columns indicate significant differences between lines $(p<0.05)$.

(A)

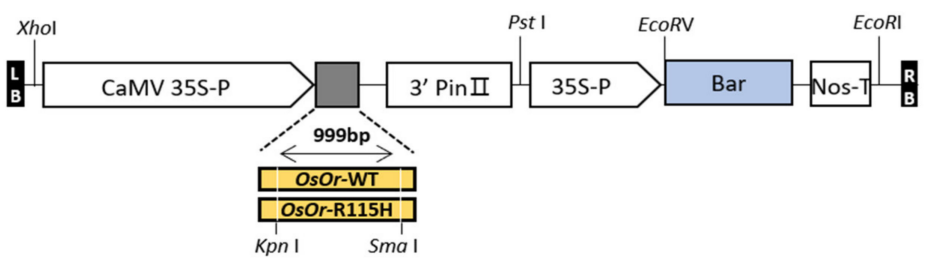

(B)
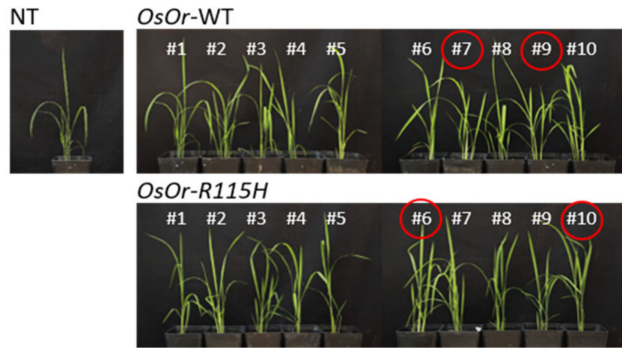

M P NT \#1 \#2 \#3 \#4 \#5 \#6 \#7) \#8\#9)\#10 OsOr-W

M P NT \#1 \#2 \#3 \#4 \#5 \#6 \#7 \#8 \#9 \#10

OsOr-R115H
(C)

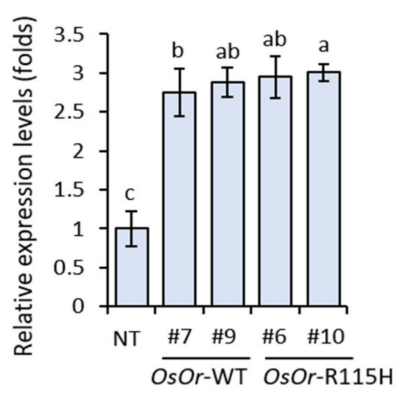

Figure 2. Generation of transgenic rice plants overexpressing $O s O r-W T$ and $O s O r-\mathrm{R} 115 \mathrm{H}$. (A) Schematic representation of the constructs used for the production of transgenic rice plants overexpressing OsOr-WT and OsOr-R115H. (B) Photographs of aerial plant parts of 1-month-old NT, OsOr-WT (\#7, \#9) and OsOr-R115H (\#6, \#10) plant lines and genotyping transgenic plants using bar-specific primers. P, positive control. (C) Transcript levels of OsOr in transgenic rice plants. Data are means \pm SE $(n=3)$. Different letters above the columns indicate significant differences between lines $(p<0.05)$. 
The expression level of OsOr gene was found to be statistically significantly higher in transgenic lines (\#7, \#9, \#6,\#10) than in NT plants (Figure 2C). In addition, the flower structure and pollen germination of transgenic plants were shown similar to those of NT plants (Figure 3A). In addition, the transgenic lines and NT plant showed similar responses to disease in the inoculation experiment with Xanthomonas oryzae pv. oryzae (Xoo) strain (Figure 3B). In order to investigate the morphological characteristics of rice grain in seed cross-sections, the results of scanning electron microscope (Quanta FEG 450, FEI Electron Microscopy) analysis showed that the seeds of the transgenic lines were almost similar to those of the NT plants (Figure 3C). As a result of examining starch content and amylose content of transgenic lines, there were no significant differences from NT plants (Figure 3D). In addition, statistical analysis showed that no significant differences were detected in transgenic lines (\#7, \#9, \#6, \#10) compared to NT plants, including shoot length, root length, seed setting rate, pollen fertility, 1000-grain weight, and yield per plant (Figure 4). Taken together, our results showed that overexpression of OsOr-WT and OsOr-R115H was not significant phenotypic alterations compared to NT plants. In addition, the carotenoid content between the transgenic lines and NT plants was analyzed using high performance liquid chromatography (HPLC) analysis. The results were almost similar in the investigated lines (Supplementary Figure S3), implying that the OsOr gene was not directly affect to the carotenoid contents in rice [33].

(A)

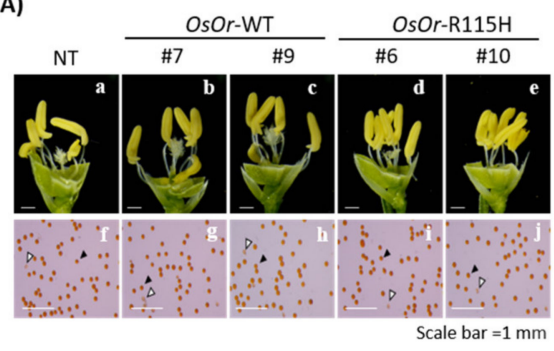

(C)

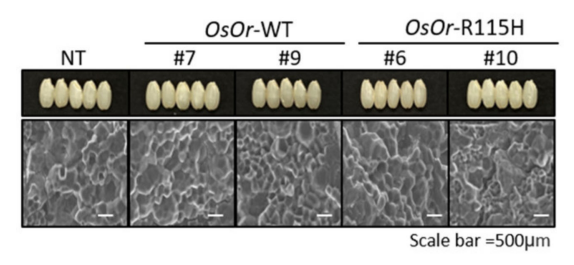

(B)
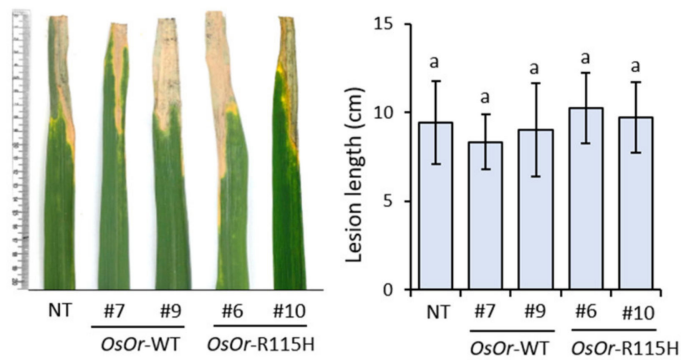

(D)
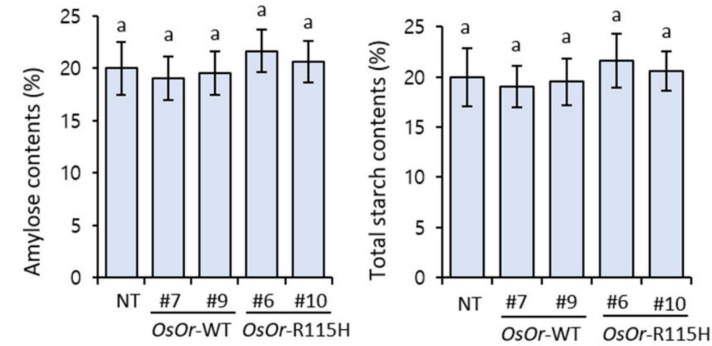

Figure 3. Phenotype of NT plants and transgenic rice lines overexpressing OsOr subjected to normal conditions. (A) Figure 7. (\#9) and OsOr-R115H (\#6, \#10) lines. (B) Lesions and lesion length of NT plants, OsOr-WT (\#7, \#9) and OsOr-R115H (\#6, \#10) lines inoculated with Xanthomonas oryzae pv. oryzae (Xoo) strain for 2 weeks. (C) Seeds morphology and SEM analysis and (D) Amylose contents (\%) and total starch contents (\%) in $\mathrm{T}_{3}$ generation. Data are means $\pm \mathrm{SE}(n=3)$. Different letters above the columns indicate significant differences between lines $(p<0.05)$. 
(A)

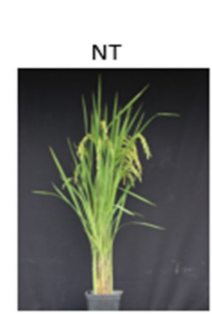

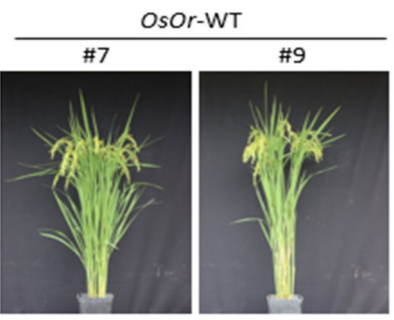

OsOr-R115H
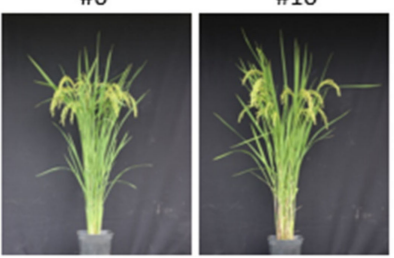

(B)
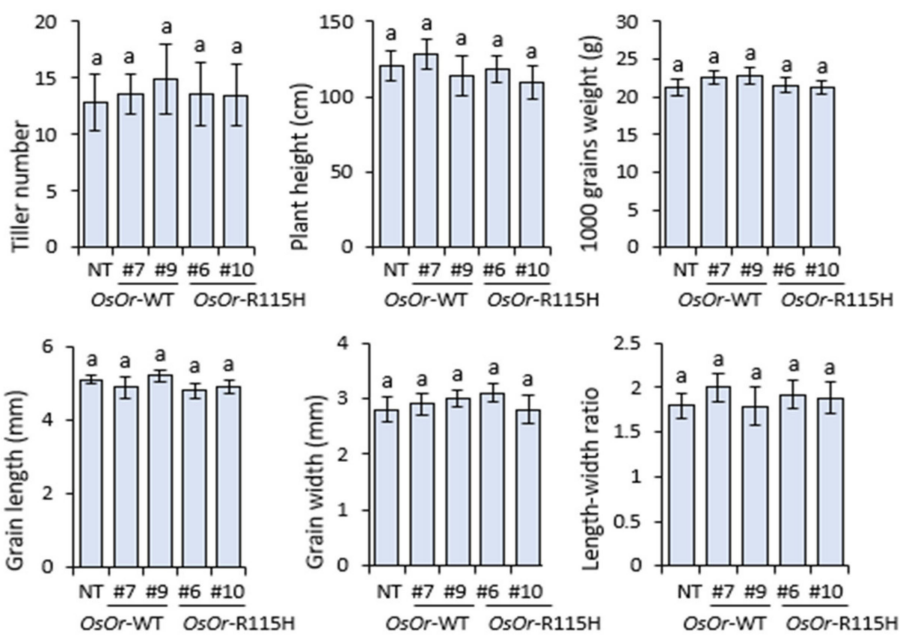

Figure 4. Agronomic traits of NT plants and transgenic rice lines overexpressing OsOr subjected to normal conditions. Morphology of mature plants (A), Tiller numbers, Plant height, 1000 grains weight, Grain length, Grain width and length width rate (B). Different letters between OsOr-WT, OsOr-R115H, and NT plants indicate significant differences $(p<0.05)$ according to Duncan test (mean $\pm \mathrm{SE}, n=3$ ).

\subsection{Overexpression of OsOr-R115H Enhances Heat Stress Tolerance}

To examine whether OsOr plays a role in heat stress tolerance, we investigated heat tolerance of the transgenic lines (OsOr-WT (\#7, \#9), and OsOr-R115H (\#6,\#10)) together with WT plants. As shown in Figure 5A, no significant morphological differences of leaf discs were observed between transgenic and NT plants before heat stress treatment. However, after incubation at $48^{\circ} \mathrm{C}$ for $24 \mathrm{~h}$, NT plants showed severe membrane damages, as evident from the dark-brown coloration of leaf discs, whereas OsOr-WT and OsOr-R115H leaf discs displayed less membrane damage. OsOr-R96H transgenic plants showed greater heat stress tolerance than NT and OsOr-WT plants (Figure 5A). Our data showed that the oxidized DAB contents of NT leaves at $12 \mathrm{~h}$ after heat stress was $21.3 \mathrm{mg} / \mathrm{g}$ DW, significantly higher than those of transgenic lines (the oxidized DAB contents of OsOr-WT \#7, \#9, OsOr-R115H \#6 and OsOr-R115H \#10 lines were 10.9, 9.2, 8.7, and 7.7, respectively) (Figure 5B). In addition, we determined the proline and chlorophyll contents in the leaves from transgenic lines and NT plants. Our results showed no difference in proline and chlorophyll contents between NT plants and transgenic lines grown under normal conditions. On the other hand, the proline and chlorophyll contents of the transgenic lines (OsOr-WT and OsOr-R115H) after heat stress were significantly higher than NT plants (Figure 6A,B). Recently, it has reported that MDA and electrolyte leakage were strongly correlated with the degree of cell membrane damage under abiotic stress [34]. Thus, we measured the MDA and REL content from transgenic and wild type plants. The data displayed no obvious difference between transgenic lines and NT plants grown under normal conditions. However, the MDA and REL contents of OsOr-WT and OsOr-R115H transgenic plants were significantly lower than NT plants grown under heat stress conditions (Figure 6C,D). As a result of analyzing carotenoids after heat stress in the transgenic plants with $\mathrm{OsOr}$-WT and $\mathrm{OsOr}-\mathrm{R} 115 \mathrm{H}$, the carotenoid content was slightly decreased compared to the results under normal conditions after $24 \mathrm{~h}$ of heat stress treatment. However, there was no difference between NT plants and overexpression plants. These results suggest that the carotenoid accumulation pathway in rice is due to differences from that of sweet potatoes. 
(A)

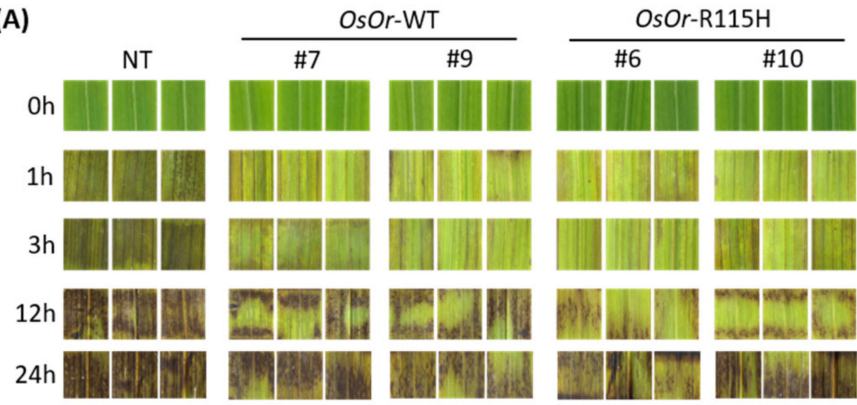

(B)

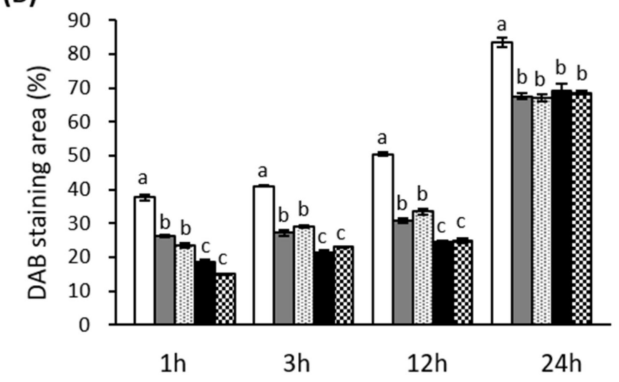

(C)

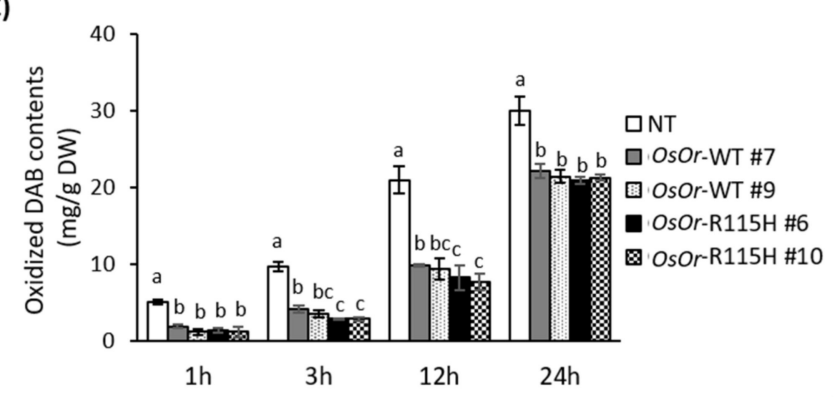

Figure 5. Analysis of heat stress tolerance in transgenic rice plants using leaf discs. (A) Images of leaf discs and quantification of ROS production in leaves using DAB staining. (B) DAB stained areas were measured and were quantified using ImageJ software in leaves at $1 \mathrm{~h}, 3 \mathrm{~h}, 12 \mathrm{~h}$ after $48^{\circ} \mathrm{C}$ heat treatment. (C) $\mathrm{H}_{2} \mathrm{O}_{2}$ production after $1 \mathrm{~h}, 3 \mathrm{~h}$ and $12 \mathrm{~h}$ of treatment with heat stress. NT, OsOr-WT and OsOr-R115H transgenic rice cell lines were treated with a DAB-HCl solution and oxidized $\mathrm{DAB}$ content was compared. Data are means $\pm \mathrm{SE}(n=3)$. Different letters above the columns indicate significant differences between lines $(p<0.05)$.

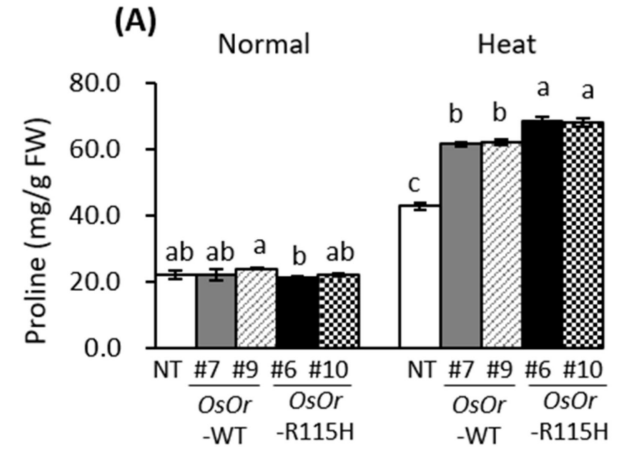

(C)

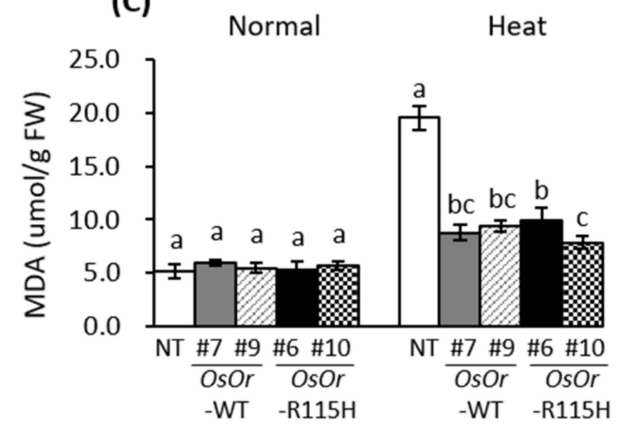

(B)

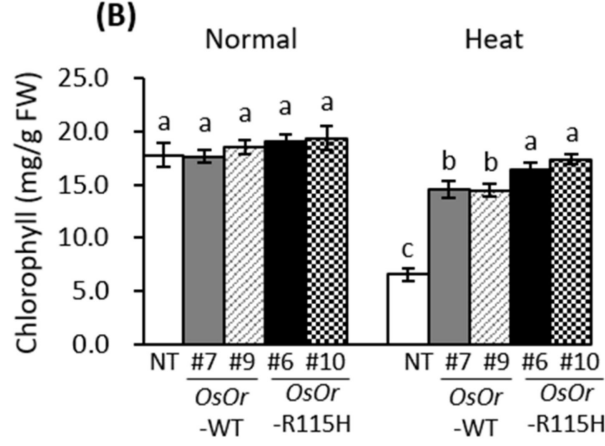

(D)

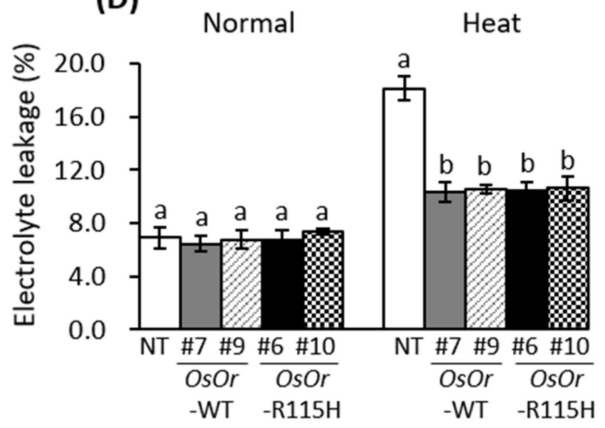

Figure 6. Contents of (A) Proline (mg/g FW), (B) chlorophyll (mg/g FW), (C) lipid peroxidation (malondialdehyde (MDA), $\mu \mathrm{mol} / \mathrm{g} \mathrm{FW}$ ) level and (D) electrolyte leakage of the NT plants and transgenic rice lines overexpressing OsOr subjected to normal and heat stress conditions. Data are means \pm SE $(n=3)$. Different letters above the columns indicate significant differences between lines $(p<0.05)$. 


\subsection{Transcription Analysis of Stress-Related Genes}

To illustrate the regulation mechanism underlying the enhanced heat stress tolerance, we measured the expression of four genes encoding ROS scavenging enzymes (OsCATA, OsCATB, OsAPX2, OsSOD-Cu/Zn) and five genes (OsLEA3, OsDREB2A, OsDREB1A, OsP5CS, $S N A C 1)$ related to abiotic stress tolerance under normal growth and heat stress conditions using qRT-PCR (Figures 7 and 8). There was no significant difference in the expression of all genes investigated under normal growth conditions. By contrast, the expression of all genes was remarkably higher in OsOr-WT and OsOr-R115H plants than in NT plants after heat stress. These results show that OsOr overexpression induces proteins involved in ROS scavenging pathways and defense mechanisms, thereby reducing oxidative damage and improving heat resistance.

(A)

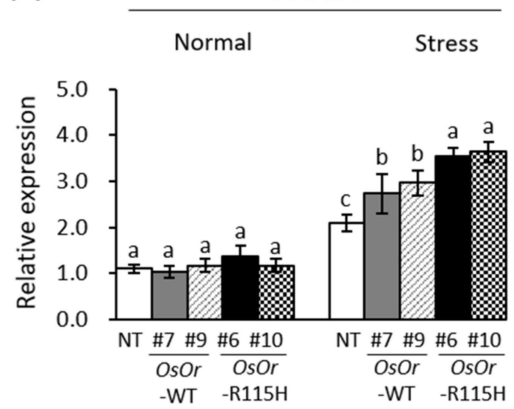

(C)
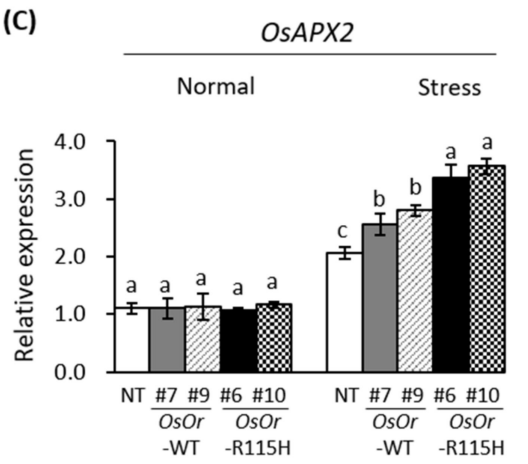

(B)
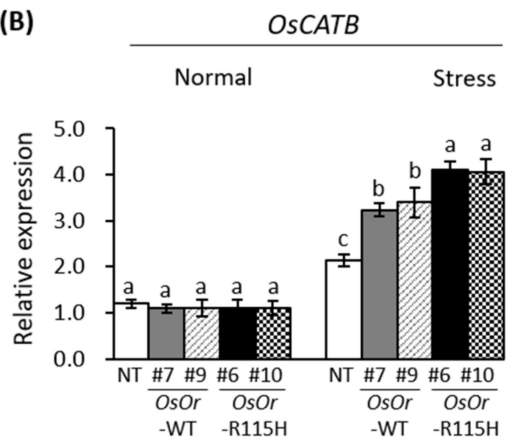

(D)
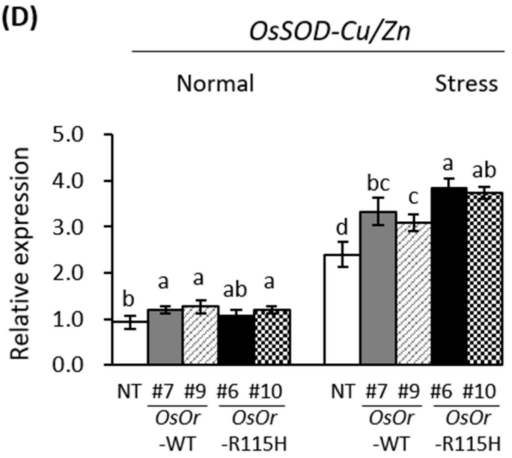

Figure 7. Expression levels of (A) OsCATA, (B) OsCATB, (C) OsAPX2, and (D) OsSOD-Cu/Zn genes in the NT plants and transgenic rice lines overexpressing OsOr subjected to normal and heat stress conditions. Data are means \pm SE $(n=3)$. Different letters above the columns indicate significant differences between lines $(p<0.05)$.

(A)

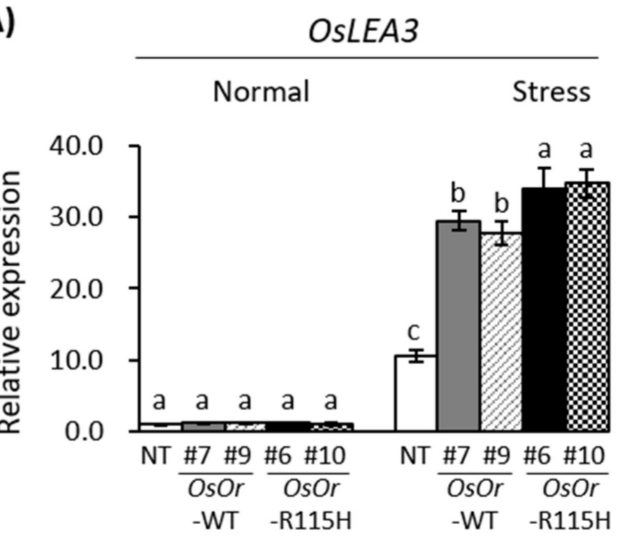

(B)

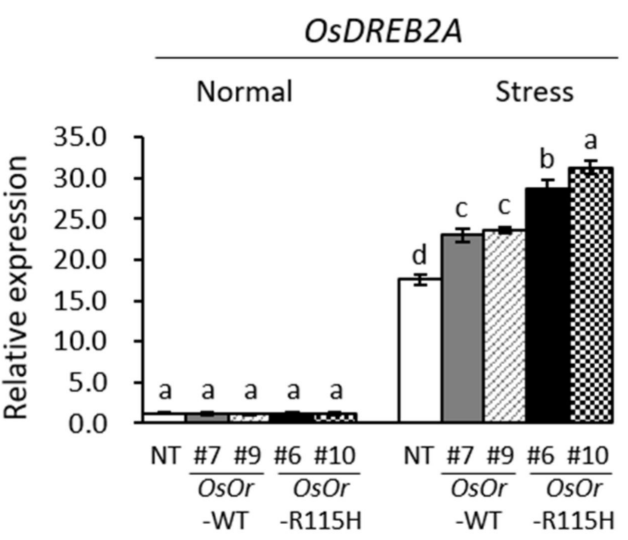

Figure 8. Cont. 
(C)

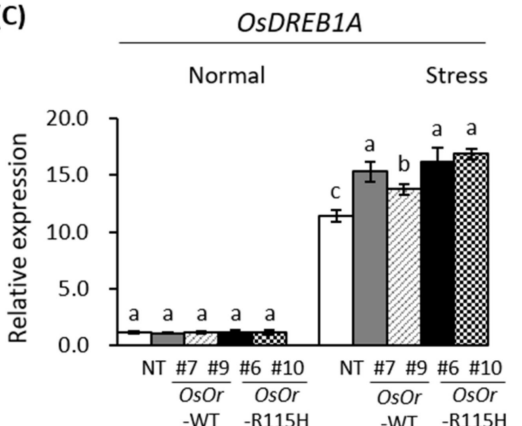

(D)

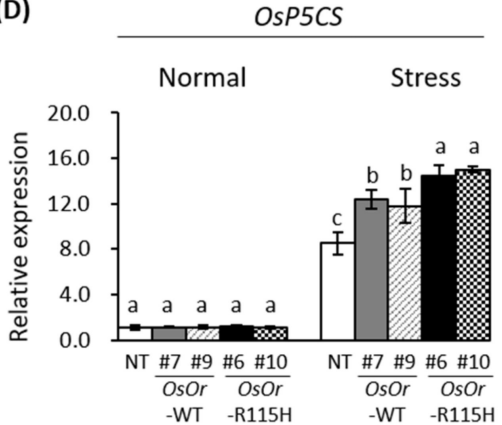

(E)

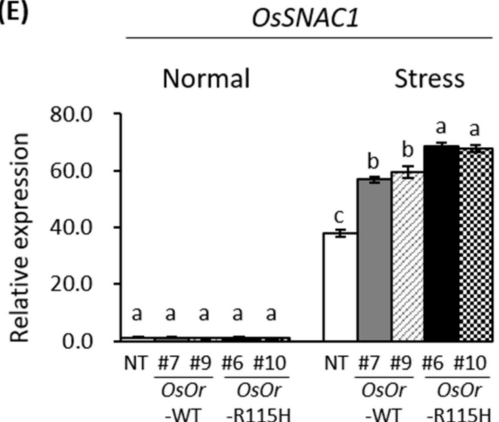

Figure 8. Expression levels of (A) OsLEA3, (B) OsDREB2A, (C) OsDREB1A, (D) OsP5CS and (E) OsSNAC1 genes in the NT and transgenic rice lines overexpressing OsOr under normal and heat stress conditions. Data are means \pm SE $(n=3)$. Different letters above the columns indicate significant differences between lines $(p<0.05)$.

\section{Discussion}

Plant orange (Or) proteins contain an N-terminal unknown region, transmembrane domains, and a C-terminal DnaJ-like domain, and meaningful progress has been made in understanding the biological function of the orange genes [8]. Some orange $(\mathrm{Or})$ genes such as $\mathrm{BoOr}$ (ABH07405), IbOr (KX792094), CmOr (A0A0D3MU35), AtOr (Q8VYD8), SIOr (NP_001315338), and DcOr (KZN00418) showed resistance to various environmental stresses as well as carotenoid accumulation [8-16]. However, the clear biological functions of these genes are still unclear and require further studies. A single nucleotide substitution in the gene encoding $\mathrm{CmOr}$ is correlated with the orange color fruit. This substitution results in an Arg to His change at the 108th amino acid of CmOr [9]. In previous report, overexpression of $\mathrm{AtOr}^{\mathrm{His}}(\mathrm{R} 90 \mathrm{H}), \mathrm{SbOr}^{\mathrm{His}}(\mathrm{R} 104 \mathrm{H})$, and $\mathrm{IbOr}^{\mathrm{His}}$ (R96H) with mutations at the site corresponding to the mutation in $\mathrm{CmOr}$ (golden SNP altering Arg to His) resulted in high total carotenoid levels and $\beta$-carotene accumulation $[13,22,35]$. In a sequence alignment, we found that OsOr possessed Arg at the 115th amino acid, corresponding to the 108th position of $\mathrm{CmOr}$, 90th position of AtOR and 96th position of IbOr. Thus, to examine whether the substitution of the conserved Arg to His in OsOr promoted carotenoid accumulation, site-directed mutagenesis of $\mathrm{OsOr}$-WT was performed to generate $\mathrm{OsOr}$ $\mathrm{R} 115 \mathrm{H}$. In this study, we report the isolation, characterization, and physiological function of the orange (Or) gene from rice. Our results show that in the overexpressing plants, OsOrWT and OsOr-R115H, carotenoid content was not changed compared to NT plants, but heat stress tolerance was enhanced. In addition, we did not find significant differences between $\mathrm{OsOr}$-WT and $\mathrm{OsOr}$-R115H. The reason why OsOr-R115H did not have a significant effect on carotenoids, unlike sweet potatoes, is thought to be due to a different mechanism of carotenoid accumulation, which is consistent with that previously reported by Yu el al. [33]. Evidence has shown that some Or genes are induced or inhibited expression by abiotic stress, and overexpression of these genes can increase the tolerance of transgenic plants to heat [8]. Similarly, our results showed rapid changes of OsOr gene expression in heat and PEG stress (Figure 1), suggesting OsOr might play a critical role in heat and PEG stress. In addition, overexpression of the golden SNP-carrying mutant $\mathrm{IbOr}$ allele was resulted to have enhanced abiotic stress tolerance as well as carotenoids accumulation [8]. In addition, Yu et al. (2021) reported that salt and cold stress resistance was reduced in Nipponbare rice plants overexpressing the OsOr gene [33]. Therefore, we constructed OsOr gene $(\mathrm{OsOr}-\mathrm{WT}$ and $\mathrm{OsOr}-\mathrm{R} 115 \mathrm{H})$ for overexpressing transgenic plants and tested their heat stress tolerance. Our data suggested that the overexpression of OsOr-WT and OsOr-R115H enhanced heat stress tolerance, and transgenic plants showed less membrane damage and aging compared to NT plants in Dongjin rice (Figures 5 and 6). In general, when plants encountered abiotic stresses such as heat and salt, REL, proline, and MDA can react quickly to survive in extreme environmental conditions $[34,36]$. Therefore, the REL, an important parameter reflecting the degree of cell membrane damage, was significantly 
lower in the leaves of the transgenic plants than in the leaves of NT plants (Figure 6D). These results suggested that the OsOr gene might protect cell membrane integrity of plants in response to heat stress. Mellacheruvu et al. [37] have reported that abiotic stress on plants causes lipid peroxidation, resulting in MDA accumulation. Our data displayed that the MDA content was higher in NT plants than in transgenic plants with OsOr-WT and OsOr-R115H (Figure 6C), which shows that heat stress has more damage to NT plants compared to transgenic plants. Furthermore, proline has been reported to act as a stabilizer to protect plants from abiotic stress damage [38]. In our data, the proline of the transgenic plant with $\mathrm{OsOr}$-WT and $\mathrm{OsOr}$-R115H accumulated more compared with NT plants under heat stress conditions. These results suggested that proline might be responsible for enhancing heat stress tolerance shown by the transgenic plants overexpression OsOr. These results showed that enhanced heat stress tolerance of the transgenic plants with OsOr-WT and $O s O r-\mathrm{R} 115 \mathrm{H}$ was at least partially related to reduced MDA content, reduced REL, and increased proline content. In addition, biochemical factors such as MDA, REL, etc. mentioned above have also contributed to significantly inducing expression of abiotic stress-response genes $[39,40]$. In our study, the expression level of four genes related to ROS-scavenging enzymes (OsCATA, OsCATB, OsAPX2 and OsSOD-Cu/Zn) and six genes in response to abiotic stress (OsLEA3, OsDREB2A, OsDREB1A, OsP5CS, SNAC1) were more highly expressed in the transgenic lines (OsOr-WT \#7, OsOr-WT \#9, OsOr-R115H \#6 and OsOr-R115H \#10) than in NT plants under heat stress conditions (Figures 7 and 8). Sirko et al. [41] reported that the function of OsCATA gene plays a role in protecting plants from damage caused by reactive oxygen species (ROS). It has been demonstrated that there is a positive correlation between the expression of OsP5CS gene and the accumulation of proline, and overexpression of OsP5CS increases resistance to abiotic stress in transgenic plants [42]. In addition, OsLEA3, OsDREB2A, OsDREB1A, and SNAC1 genes were reported to be sensitive to abiotic stress [43-47]. It was reported that the plant hormone ABA is produced by five 9-cis-epoxycarotenoid dioxygenases (NCEDs), and strigolactones are synthesized by the enzymes CCD7 and CCD8 [48]. However, because of examining the expression of OsOr gene, it was slightly upregulated by ABA stress, and there was no difference in carotenoid content in transgenic plants. Taken together, the results showed that the transgenic lines further enhanced heat stress tolerance, perhaps by strengthening the expression levels of genes related to ROS-scavenging enzymes and responding to abiotic stress under heat stress conditions. The current study also demonstrated that the OsOr gene could serve as a candidate for breeding heat stress tolerant varieties in rice. Future research should further explore OsOr gene regulatory network and functionality using CRISPR/Cas9 system mediated base-editing technique, etc., in greater depth to unravel the mechanism for controlling heat stress tolerance in rice.

Supplementary Materials: The following are available online at https:/ / www.mdpi.com/article/10 .3390/genes12121891/s1, Figure S1: (A) Gene structure of Or gene (Os02g0651300) in rice. (B) Nucleotide and amino acid sequences of $\mathrm{Or}$ gene in rice. (C) Multiple sequence alignments of OsOr proteins identified. Brassica oleracea (BoOr; ABH07405), Arabidopsis thaliana (AtOr; AT5G06130), Daucus carota (DcOr; DCAR_009172), Cucumis melo (CmOr; MELO3C005449.2), Ipomoea batatas (IbOr; itb01g14720), Solanum lycopersicum (SlOr; Solyc03g093830). Figure S2: TaqMan PCR analysis for copy number assays using TaqMan probe for single copy selection in T0 transgenic Rice. +; single copy, -; multi copy, $\mathrm{T}_{3}$-homozygous and $\mathrm{T}_{3}$-heterozygous; single copy control, NC; negative control, NT; Non-transgenic control. Figure S3: Contents and composition of carotenoids in the leaves of Or gene transgenic plants relative to NT plants in normal condition(A) and $24 \mathrm{~h}$ heat stress condition(B). Carotenoid levels determined by HPLC analysis of leaf tissues harvested from three independent transgenic plants for each construct. Data represent mean \pm SD of three independent replicates $(p<0.05$; One-way ANOVA, followed by Duncan's post hoc test). Table S1: Primers used for qRT-PCR analysis in this study. 
Author Contributions: Formal analysis, Y.J.J., J.Y.G. and H.J.L.; methodology, J.S.P., J.Y.K. and Y.J.L.; investigation, M.-J.A., M.-S.K., and Y.-G.C.; resources, M.-J.A., S.-S.K., and H.S.K.; software, K.K.K.; supervision, K.K.K.; writing-original draft, Y.J.J. and J.Y.G.; writing-review and editing, K.K.K. All authors read and approved the manuscript. All authors have read and agreed to the published version of the manuscript.

Funding: This work was carried out with the support of "Cooperative Research Program for Agriculture Science \& Technology Development (PJ01477604)" Rural Development Administration (RDA) and basic science research program through the National Research Foundation of Korea (NRF) funded by the ministry education (2021R1I1A4A01057295) Republic of Korea.

Institutional Review Board Statement: Not applicable.

Informed Consent Statement: Not applicable.

Data Availability Statement: Not applicable.

Conflicts of Interest: The authors declare no conflict of interest. The funders had no role in the design of the study; in the collection, analyses, or interpretation of data; in the writing of the manuscript, or in the decision to publish the results.

\section{References}

1. Iba, K. Acclimative response to temperature stress in higher plants: Approaches of gene engineering for temperature tolerance. Annu. Rev. Plant Biol. 2002, 53, 225-245. [CrossRef]

2. Choudhury, F.K.; Rivero, R.M.; Blumwald, E.; Mittler, R. Reactive oxygen species, abiotic stress and stress combination. Plant J. 2017, 90, 856-867. [CrossRef]

3. Vollenweider, P.; Günthardt-Goerg, M.S. Diagnosis of abiotic and biotic stress factors using the visible symptoms in foliage. Environ. Pollut. 2005, 137, 455-465. [CrossRef]

4. Loeffler, C.; Berger, S.; Guy, A.; Durand, T.; Bringmann, G.; Dreyer, M.; Mueller, M.J. B 1 -phytoprostanes trigger plant defense and detoxification responses. Plant Physiol. 2005, 137, 328-340. [CrossRef]

5. Mueller, S.; Hilbert, B.; Dueckershoff, K.; Roitsch, T.; Krischke, M.; Mueller, M.J.; Berger, S. General detoxification and stress responses are mediated by oxidized lipids through TGA transcription factors in Arabidopsis. Plant Cell 2008, 20, 768-785. [CrossRef]

6. Raja, V.; Majeed, U.; Kang, H.; Andrabi, K.I.; John, R. Abiotic stress: Interplay between ROS, hormones and MAPKs. Environ. Exp. Bot. 2017, 137, 142-157. [CrossRef]

7. Zhang, M.; Su, J.; Zhang, Y.; Xu, J.; Zhang, S. Conveying endogenous and exogenous signals: MAPK cascades in plant growth and defense. Curr. Opin. Plant Biol. 2018, 45, 1-10. [CrossRef]

8. Lu, S.; Van Eck, J.; Zhou, X.; Lopez, A.B.; O'Halloran, D.M.; Cosman, K.M.; Li, L. The cauliflower Or gene encodes a DnaJ cysteine-rich domain-containing protein that mediates high levels of $\beta$-carotene accumulation. Plant Cell 2006, 18, 3594-3605. [CrossRef] [PubMed]

9. Tzuri, G.; Zhou, X.; Chayut, N.; Yuan, H.; Portnoy, V.; Meir, A.; Tadmor, Y.A. 'golden'SNP in CmOr governs the fruit flesh color of melon (Cucumis melo). Plant J. 2015, 82, 267-279. [CrossRef] [PubMed]

10. Chayut, N.; Yuan, H.; Ohali, S.; Meir, A.; Sa'ar, U.; Tzuri, G.; Tadmor, Y. Distinct mechanisms of the ORANGE protein in controlling carotenoid flux. Plant Physiol. 2017, 173, 376-389. [CrossRef] [PubMed]

11. Lopez, A.B.; Van Eck, J.; Conlin, B.J.; Paolillo, D.J.; O’Neill, J.; Li, L. Effect of the cauliflower Or transgene on carotenoid accumulation and chromoplast formation in transgenic potato tubers. J. Exp. Bot. 2008, 59, 213-223.89. [CrossRef] [PubMed]

12. Zhou, X.; Welsch, R.; Yang, Y.; Álvarez, D.; Riediger, M.; Yuan, H.; Li, L. Arabidopsis OR proteins are the major posttranscriptional regulators of phytoene synthase in controlling carotenoid biosynthesis. Proc. Natl. Acad. Sci. USA 2015, 112, 3558-3563. [CrossRef] [PubMed]

13. Yuan, H.; Owsiany, K.; Sheeja, T.E.; Zhou, X.; Rodriguez, C.; Li, Y.; Li, L. A single amino acid substitution in an ORANGE protein promotes carotenoid overaccumulation in Arabidopsis. Plant Physiol. 2015, 169, 421-431. [CrossRef]

14. Park, S.C.; Kim, S.H.; Park, S.; Lee, H.U.; Lee, J.S.; Park, W.S.; Kwak, S.S. Enhanced accumulation of carotenoids in sweetpotato plants overexpressing IbOr-Ins gene in purple-fleshed sweetpotato cultivar. Plant Physiol. Biochem. 2015, 86, 82-90. [CrossRef]

15. Wang, Z.; Ke, Q.; Kim, M.D.; Kim, S.H.; Ji, C.Y.; Jeong, J.C.; Kwak, S.S. Transgenic alfalfa plants expressing the sweetpotato Orange gene exhibit enhanced abiotic stress tolerance. PLOS ONE 2015, 10, e0126050. [CrossRef] [PubMed]

16. Kim, H.S.; Ji, C.Y.; Lee, C.J.; Kim, S.E.; Park, S.C.; Kwak, S.S. Orange: A target gene for regulating carotenoid homeostasis and increasing plant tolerance to environmental stress in marginal lands. J. Exp. Bot. 2018, 69, 3393-3400. [CrossRef]

17. Park, S.; Kim, H.S.; Jung, Y.J.; Kim, S.H.; Ji, C.Y.; Wang, Z.; Kwak, S.S. Orange protein has a role in phytoene synthase stabilization in sweetpotato. Sci. Rep. 2016, 6, 1-12. [CrossRef]

18. Kang, L.; Kim, H.S.; Kwon, Y.S.; Ke, Q.; Ji, C.Y.; Park, S.C.; Kwak, S.S. IbOr regulates photosynthesis under heat stress by stabilizing IbPsbP in sweetpotato. Front. Plant Sci. 2017, 8, 989. [CrossRef] 
19. Yazdani, M.; Sun, Z.; Yuan, H.; Zeng, S.; Thannhauser, T.W.; Vrebalov, J.; Li, L. Ectopic expression of ORANGE promotes carotenoid accumulation and fruit development in tomato. Plant Biotechnol. J. 2019, 17, 33-49. [CrossRef] [PubMed]

20. Ellison, S.L.; Luby, C.H.; Corak, K.E.; Coe, K.M.; Senalik, D.; Iorizzo, M.; Dawson, J.C. Carotenoid presence is associated with the Or gene in domesticated carrot. Genetics 2018, 210, 1497-1508. [CrossRef]

21. Kim, S.E.; Kim, H.S.; Wang, Z.; Ke, Q.; Lee, C.J.; Park, S.U.; Kwak, S.S. A single amino acid change at position 96 (Arg to His) of the sweetpotato Orange protein leads to carotenoid overaccumulation. Plant Cell Rep. 2019, 38, 1393-1402. [CrossRef]

22. Kim, S.H.; Ahn, Y.O.; Ahn, M.J.; Lee, H.S.; Kwak, S.S. Down-regulation of $\beta$-carotene hydroxylase increases $\beta$-carotene and total carotenoids enhancing salt stress tolerance in transgenic cultured cells of sweetpotato. Phytochemistry 2012, 74, 69-78. [CrossRef] [PubMed]

23. Jung, Y.J.; Lee, H.J.; Kim, J.H.; Kim, D.H.; Kim, H.K.; Cho, Y.G.; Kang, K.K. CRISPR/Cas9-targeted mutagenesis of F3' H, DFR and LDOX, genes related to anthocyanin biosynthesis in black rice (Oryza sativa L.). Plant Biotechnol. Rep. 2019, 13, 521-531. [CrossRef]

24. Lee, H.J.; Abdula, S.E.; Jee, M.G.; Jang, D.W.; Cho, Y.G. High-efficiency and Rapid Agrobacterium-mediated genetic transformation method using germinating rice seeds. J. Plant Biotechnol. 2011, 38, 251-257. [CrossRef]

25. Shi, W.; Li, X.; Schmidt, R.C.; Struik, P.C.; Yin, X.; Jagadish, S.K. Pollen germination and in vivo fertilization in response to high-temperature during flowering in hybrid and inbred rice. Plant Cell Environ. 2018, 41, 1287-1297. [CrossRef] [PubMed]

26. Song, W.Y.; Wang, G.L.; Chen, L.L.; Kim, H.S.; Pi, L.Y.; Holsten, T.; Ronald, P. A receptor kinase-like protein encoded by the rice disease resistance gene, Xa21. Science 1995, 270, 1804-1806. [CrossRef] [PubMed]

27. Chern, M.; Canlas, P.E.; Fitzgerald, H.A.; Ronald, P.C. Rice NRR, a negative regulator of disease resistance, interacts with Arabidopsis NPR1 and rice NH1. Plant J. 2005, 43, 623-635. [CrossRef]

28. Lim, C.J.; Kim, H.Y.; Lee, C.H.; Kim, Y.U.; Back, K.W.; Bae, J.M.; Ahn, M.J. Variation in carotenoid composition in carrots during storage and cooking. PNF 2009, 14, 240-245. [CrossRef]

29. Cai, W.; Liu, W.; Wang, W.S.; Fu, Z.W.; Han, T.T.; Lu, Y.T. Overexpression of rat neurons nitric oxide synthase in rice enhances drought and salt tolerance. PLoS ONE 2015, 10, e0131599.

30. Tang, Y.; Liu, K.; Zhang, J.; Li, X.; Xu, K.; Zhang, Y.; Li, C. JcDREB2, a physic nut AP2/ERF gene, alters plant growth and salinity stress responses in transgenic rice. Front. Plant Sci. 2017, 8, 306.

31. Xia, Z.; Xu, Z.; Wei, Y.; Wang, M. Overexpression of the maize sulfite oxidase increases sulfate and GSH levels and enhances drought tolerance in transgenic tobacco. Front. Plant Sci. 2018, 9, 298.

32. Duncan, D.B. Multiple range and multiple $F$ tests. Biometrics 1955, 11, 1-42. [CrossRef]

33. Yu, Y.; Yu, J.; Wang, Q.; Wang, J.; Zhao, G.; Wu, H.; Fang, J. Overexpression of the rice ORANGE gene OsOR negatively regulates carotenoid accumulation, leads to higher tiller numbers and decreases stress tolerance in Nipponbare rice. Plant Sci. 2021, 310, 110962. [CrossRef] [PubMed]

34. James, D.; Borphukan, B.; Fartyal, D.; Ram, B.; Singh, J.; Manna, M.; Reddy, M.K. Concurrent overexpression of OsGS1; 1 and OsGS2 genes in transgenic rice (Oryza sativa L.): Impact on tolerance to abiotic stresses. Front. Plant Sci. 2018, 9, 786. [CrossRef]

35. Kim, S.E.; Lee, C.J.; Park, S.U.; Lim, Y.H.; Park, W.S.; Kim, H.J.; Ahn, M.J.; Kwak, S.S.; Kim, H.S. Overexpression of the Golden SNP-Carrying Orange Gene Enhances Carotenoid Accumulation and Heat Stress Tolerance in Sweetpotato Plants. Antioxidants 2021, 10, 51. [CrossRef]

36. Wei, Q.; Luo, Q.; Wang, R.; Zhang, F.; He, Y.; Zhang, Y.; He, G. A wheat R2R3-type MYB transcription factor TaODORANT1 positively regulates drought and salt stress responses in transgenic tobacco plants. Front. Plant Sci. 2017, 8, 1374.

37. Mellacheruvu, S.; Tamirisa, S.; Vudem, D.R.; Khareedu, V.R. Pigeonpea hybrid-proline-rich protein (CcHyPRP) confers biotic and abiotic stress tolerance in transgenic rice. Front. Plant Sci. 2016, 6, 1167. [CrossRef]

38. Rejeb, B.; Abdelly, C.; Savouré, A. Proline, a multifunctional amino-acid involved in plant adaptation to environmental constraints. Biol. Aujourd'hui 2012, 206, 291-299. [CrossRef]

39. Zhu, N.; Cheng, S.; Liu, X.; Du, H.; Dai, M.; Zhou, D.X.; Zhao, Y. The R2R3-type MYB gene OsMYB91 has a function in coordinating plant growth and salt stress tolerance in rice. Plant Sci. 2015, 236, 146-156. [CrossRef]

40. Gu, M.; Zhang, J.; Li, H.; Meng, D.; Li, R.; Dai, X.; Xu, G. Maintenance of phosphate homeostasis and root development are coordinately regulated by MYB1, an R2R3-type MYB transcription factor in rice. J. Exp. Bot. 2017, 68, 3603-3615. [CrossRef]

41. Sirko, A.; Błaszczyk, A.; Liszewska, F.; Kazimierczuk, K.; Gaganidze, D. Genetic engineering of oxidative stress resistance in plants. In Sulphur in Plants; Springer: Dordrecht, The Netherlands, 2003; pp. 245-263.

42. Jacobs, M.; Angenon, G.; Hermans, C.; Thu, T.T.; Roosens, N.H. Proline accumulation and $\Delta^{1}$-pyrroline-5-carboxylate synthetase gene properties in three rice cultivars differing in salinity and drought tolerance. Plant Sci. 2003, 165, 1059-1068.

43. Dubouzet, J.G.; Sakuma, Y.; Ito, Y.; Kasuga, M.; Dubouzet, E.G.; Miura, S.; Yamaguchi-Shinozaki, K. OsDREB genes in rice, Oryza sativa L., encode transcription activators that function in drought-, high-salt-and cold-responsive gene expression. Plant J. 2003, 33, 751-763. [CrossRef] [PubMed]

44. Hu, H.; Dai, M.; Yao, J.; Xiao, B.; Li, X.; Zhang, Q.; Xiong, L. Overexpressing a NAM, ATAF, and CUC (NAC) transcription factor enhances drought resistance and salt tolerance in rice. Proc. Natl. Acad. Sci. USA 2006, 103, 12987-12992. [CrossRef] [PubMed]

45. $\mathrm{Hu}, \mathrm{T} . Z$. OsLEA3, a late embryogenesis abundant protein gene from rice, confers tolerance to water deficit and salt stress to transgenic rice. Russ. J. Plant Physiol. 2008, 55, 530-537. [CrossRef]

46. Mallikarjuna, G.; Mallikarjuna, K.; Reddy, M.K.; Kaul, T. Expression of OsDREB2A transcription factor confers enhanced dehydration and salt stress tolerance in rice (Oryza sativa L.). Biotechnol. Lett. 2011, 33, 1689-1697. [CrossRef] [PubMed] 
47. Liu, G.; Li, X.; Jin, S.; Liu, X.; Zhu, L.; Nie, Y.; Zhang, X. Overexpression of rice NAC gene SNAC1 improves drought and salt tolerance by enhancing root development and reducing transpiration rate in transgenic cotton. PLOS ONE 2014, 9, e86895. [CrossRef] [PubMed]

48. Auldridge, M.E.; McCarty, D.R.; Klee, H.J. Plant carotenoid cleavage oxygenases and their apocarotenoid products. Curr. Opin. Plant Biol. 2006, 9, 315-321. [CrossRef] 